\title{
PROPORÇÃO DE BACTÉRIAS MULTIRRESISTENTES DE UM HOSPITAL PÚBLICO SUL-BRASILEIRO
}

\author{
MULTI-DRUG RESISTANT ORGANISMS RATIO OF A PUBLIC HOSPITAL IN \\ SOUTHERN BRAZIL
}

DOI: $10.5380 /$ rmu.v1i1.40679

Bernardo Montesanti Machado de Almeida', Giovanni Luis Breda', Monica Gomes da Silva'

\section{RESUMO}

Introdução: O conhecimento da epidemiologia de bactérias em instituições hospitalares é de fundamental importância para guiar o tratamento empírico das infecções relacionadas aos cuidados de saúde. Objetivos: Analisar o perfil de sensibilidade das culturas realizadas em diferentes sítios dos principais patógenos multirresistentes no Hospital de Clínicas-UFPR, Curitiba, Brasil. Metodologia: Os dados foram analisados a partir da planilha de culturas do Serviço de Controle de Infecção Hospitalar (SCIH) do Hospital de Clínicas. Resultados: Foram analisadas 2312 amostras. Das 304 amostras de S. aureus, MRSA representou 18,4\%. Dos isolados de Enterococcus sp, 48,1\% eram VRE, em sua maioria representado pelo E. faecium. Porém a proporção de VRE em hemoculturas foi de $9,5 \%$. De 731 amostras de Enterobactérias, $41 \%$ eram resistentes ao cefepima. Klebsiella pneumoniae foi a que apresentou maior proporção de cepas resistentes ao cefepima $(65,9 \%)$ entre as Enterobactérias. As amostras de Acinetobacter baumannii apresentaram altas taxas de resistência à ampicilina-sulbactam (89,2\%) e aos carbapenêmicos (90,3\%). Dos antimicrobianos avaliados, o que apresentou melhor cobertura para os isolados de Pseudomonas aeruginosa foi a ceftazidima $(79,2 \%)$. Conclusão: A proporção de MRSA foi menor do que a de outros hospitais brasileiros. A alta prevalência de Enterobactérias e de gram negativos não fermentadores de glicose resistentes confirmam a tendência nacional e latino-americana, com proporções maiores que a de hospitais norte americanos e europeus.

Palavras-Chave: Epidemiologia, Sensibilidade, Organismos Multi-droga resistentes

\section{ABSTRACT}

Introduction: It is important to know the local prevalence of multidrug resistance bacterias in hospital environment in order to adequate empirical choice of antimicrobial therapy. Objectives: Describe antimicrobial activity to more frequent isolates from different sites of cultures in a tertiary hospital (Hospital de Clinicas - UFPR, Curitiba, Brazil). Methods: Information was analyzed from the culture data, provided by Service of Infection Control ( $\mathrm{SClH}$ ) at Hospital de Clínicas. Results: A total of 2312 samples were analyzed. Of the 304 samples of S. aureus, MRSA accounted for $18.4 \%$. Of Enterococcus sp, $48.1 \%$ were VRE, mostly represented by E. faecium. In contrast, the proportion of VRE in blood cultures was only $9.5 \%$. Of the 731 cultures of Enterobacteriaceae, $41 \%$ were resistant to cefepima. Klebsiella pneumoniae showed the highest proportion of strains resistant to cefepime (65.9\%) among the Enterobacteriaceae. Samples of Acinetobacter baumannii showed high levels of resistance to ampicillin-sulbactam (89.2\%) and carbapenems (90.3\%). Ceftazidime showed the better coverage $(79,2 \%)$ between the antibiotic analyzed for isolates of Pseudomonas aeruginosa (79.2\%). Conclusions: The proportion of MRSA was lower than that of other Brazilian hospitals. The high prevalence of resistant Enterobacteriaceae and gram-negative non-fermenting glucose confirm the national and Latin America trend, with the largest proportions than North American and European hospitals.

Key-words: Epidemiology. Sensibility. Multi-drug resistant organisms

1-Universidade Federal do Paraná

Contato do Autor / Mail to:

Bernardo Montesanti Machado de Almeida - machado_de_almeida@hotmail.com

Rua General Carneiro, 181 - Alto da Glória Curitiba - PR, 80060-900, 3ํ Andar, Serviço de Infectologia-HC/UFPR 


\section{INTRODUÇÃO}

A prescrição de antimicrobianos é prática comum no ambiente hospitalar. Há evidências de aumento progressivo no uso de antibióticos e de que um terço das prescrições não estão de acordo com os as diretrizes vigentes ${ }^{1}$. Seu uso indiscriminado é um dos fatores responsáveis pela emergência de germes multirresistentes, que estão associados a aumento da morbimortalidade dentro do ambiente hospitalar ${ }^{2}$. 0 aumento crescente da resistência a diferentes classes de antibióticos faz com que a escolha empírica dos tratamentos se torne um desafio. Por outro lado, o atraso no início da antibioticoterapia está associado a aumento na mortalidade ${ }^{3}$.

Dentre as bactérias gram positivas multirresistentes frequentemente isoladas, o $\mathrm{S}$. aureus é agente etiológico frequentemente implicado na ocorrência de infecção de pele e partes moles, pneumonia nosocomial, especialmente na pneumonia associado à ventilação mecânica (PAV), e infecção de corrente sanguínea ${ }^{4}$. Dados brasileiros mostram que o S. aureus resistente à meticilina (MRSA) corresponde a $30-60 \%$ dos isolados em alguns hospitais ${ }^{5}$. O clone BECMRSA é um dos mais prevalentes no Brasil e é caracteristicamente resistente a outras classes de antimicrobianos como clindamicina, eritromicina e sulfametoxazol-trimetropim. Outros clones não BECMRSA estão emergindo nos últimos anos. Um exemplo são os clones que contém o gene SCCmec tipo IV, que geralmente são sensíveis às demais classes ${ }^{6}$. 0 Enterococcus $s p$ é outro patógeno gram positivo de interesse médico, devido ao aumento na incidência de cepas resistentes à vancomicina (VRE). Dados latinoamericanos mostram que houve aumento na incidência de VRE de 5\% para 15,5\% entre 2003 e $2008 .^{7}$

Em relação aos gram negativos, há maior representatividade de patógenos produtores de $\beta$ lactamase de espectro estendido (ESBL) e de não fermentadores de glicose multirresistentes, como Pseudomonas aeruginosa e Acinetobacter baumanni, quando comparado com dados europeus e americanos ${ }^{6}$. Os patógenos produtores de ESBL representam até 50\% dos isolados de Klebsiella pneumoniae em hospitais brasileiros. Variantes produtores de CTX-M, uma das ESBL, são as mais prevalentes, porém são escassos os dados nacionais a respeito das características moleculares das ESBL. Pesudomonas aeruginosa e Acinetobacter baumannii são importantes agentes etiológicos de infecções hospitalares e têm motivado o uso empírico de polimixina B e colistina em algumas unidades de terapia intensiva (UTI) brasileiras ${ }^{6}$. Há vários mecanismos de resistência implicados na resistência da Pseudomonas aeruginosa, como bombas de efluxo e perda de porinas, além de hiperprodução de ampC, e produção de $\beta$-lactamases como ESBL e metalo$\beta$-lactamases ${ }^{8}$. Os principais mecanismos de resistência no caso do Acinetobacter baumannii são por produção de $\beta$-lactamase OXA-23 e OXA-143. Isolados brasileiros mostram resistência aos carbapenêmicos que variam de $25 \%$ a $45 \%$. A grande variedade e coexistência de diferentes mecanismos de ação entre os gram negativos torna a escolha empírica um desafio crescente.

Por isso, faz-se necessária a monitorização e divulgação rotineira do perfil de sensibilidade dos patógenos mais prevalentes no ambiente hospitalar com o intuito de maximizar o acerto na escolha de terapia empírica das infecções associados aos cuidados de saúde.

\section{OBJETIVOS}

O estudo tem por objetivo a avaliação do perfil de sensibilidade dos principais patógenos causadores de infecção associado aos cuidados de saúde (S. aureus, Enterobactérias, Pseudomonas aeruginosa e Acinetobacter baumanii) nos diferentes sítios de coleta (sangue, amostras respiratórias e urina) no Hospital de Clínicas da Universidade Federal do Paraná.

\section{METODOLOGIA}

Os dados foram extraídos da planilha de culturas fornecida pelo Serviço de Controle de Infecção Hospitalar do Hospital de Clínicas, que contém os resultados de todas as culturas realizadas pelo laboratório do hospital no período de 10 meses, correspondentes a janeiro de 2012 a outubro de 2012 . Foram avaliados somente amostras com resultado de antibiograma disponível. Foram excluídas as amostras do mesmo paciente com isolamento do mesmo patógeno.

\section{RESULTADOS}

Foram analisadas 2312 culturas de 1151 pacien-tes. Hemoculturas representaram $12,27 \%$ das amostras e desses, 59,9\% (236 amostras) eram gram positivos. Enterobactérias foram responsáveis por $32,23 \%$ das bacteremias, sendo que a Klebsiella sp foi o principal patógeno atrás do ENPC, superando o $S$. aureus.

\section{Gram Positivos}

Entre os gram positivos, foram analisadas as ocorrências de Staphylococcus aureus, estafilococos não produtores de coagulase (ENPC) e Enterococcus $s p$.

Foram isolados 304 amostras de S. aureus, sendo 56 $(18,4 \%)$ de hemocultura. Conforme representado na figura 1, verificou-se que $67(22 \%)$ eram cepas resistentes à oxacilina. Dentre as hemoculturas, 9 (16\%) eram MRSA. Dentre as cepas resistentes à oxacilina, 68 
$(94,1 \%)$ do total e $9(100 \%)$ das hemoculturas foram sensíveis ao sulfametoxazol-trimetoprim.

\begin{tabular}{cc}
\hline Patógeno & Isolados (\%) \\
\hline ENPC & $154(39,09)$ \\
\hline Klebisella sp & $43(10,91)$ \\
S. aureus & $39(9,9)$ \\
Enterococcus sp & $32(8,12)$ \\
E. coli & $29(7,36)$ \\
Pseudomonas sp & $20(5,08)$ \\
Enterobacter sp & $18(4,57)$ \\
\hline Acinetobacter sp & $16(4,06)$ \\
S. viridans & $10(2,54)$ \\
Serratia $s p$ & $8(2,03)$ \\
Citrobacter $s p$ & $6(1,52)$ \\
Morganella $s p$ & $2(0,51)$ \\
\hline outros & $17(4,31)$ \\
\hline
\end{tabular}

Tabela 1: Isolados em Hemoculturas.

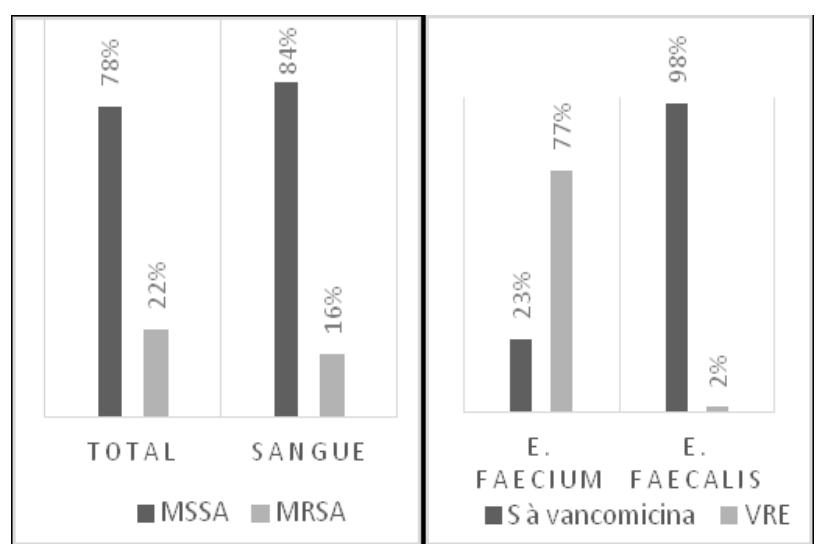

Figura 1: proporção de MRSA dentre todos os isolados de $S$. aureus e entre as hemoculturas; B: proporção de VRE entre os isolados de E. faecium e E. faecalis

Dentre os ENPC, 293 (76,7\%) eram resistentes à oxacilina em relação ao total. Nas hemoculturas foram encontrados $179(80,3 \%)$ isolados resistentes. Dos 322 isolados de Enterococcus sp, 120 (37,3\%) eram representados por E. faecalis e 198 (61,5\%) por $E$. faecium. Do total, $155(48,1 \%)$ eram VRE. Entre $E$. faecalis, cepas resistentes à vancomicina representaram apenas $1,7 \%$, enquanto que entre $E$. faecium, representaram 76,8\%. Quando excluído amostras de swab retal, realizado para fins de vigilância epidemiológica, a proporção de VRE foi de 11,17\% em relação ao total e de $28,13 \%$ dentre os Enterococcus faecium Quando avaliados apenas as hemoculturas, 38 $(90,5 \%)$ eram sensíveis à vancomicina e apenas $4(9,5 \%)$ eram VRE, sendo 3 E. faecium e 1 E. gallinarum.
Dentre os gram negativos, foram analisados Escherichia coli, Klebsiella pneumoniae, Acinetobacter baumannii e Pseudomonas aeruginosa. Foram avaliados 731 isolados de enterobactérias, sendo que (41\%) eram resistentes à cefalosporina de quarta geração, mostrando a alta prevalência de patógenos produ-tores de ESBL. A enterobactéria mais frequentemente isolada foi a E.coli $(52,6 \%)$. A resistência desse patógeno à cefepima ocorreu em 17,2\%, 16,4\% e 19,6\% nas hemoculturas, urina e no total, respectivamente. Klebsiella pneumoniae foi a bactéria mais prevalente em hemoculturas $(40,3 \%)$ dentre as enterobactérias. Cepas resistentes à cefalosporina de quarta geração representaram $60,4 \%$ das hemoculturas, $68,5 \%$ das uroculturas e $65,9 \%$ do total.

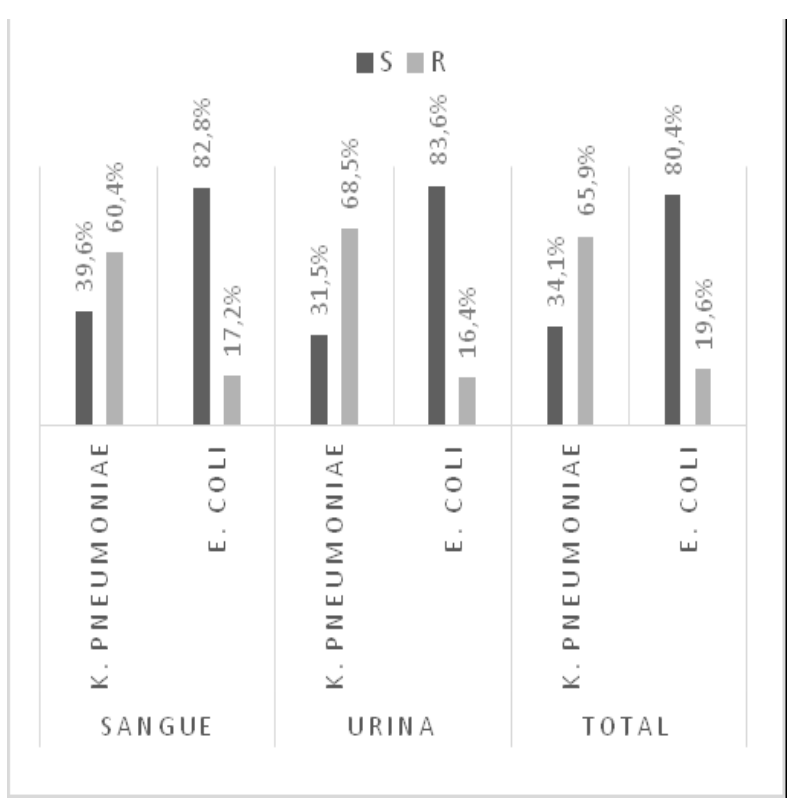

Figura 2: resistência de K. pneumoniae e E. coli ao cefepima em culturas de diferentes sítios.

Os isolados de Acinetobacter baumannii apresen-taram altas taxas de resistência à ampicilina-sulbactam e ao meropenem $(89,2 \%$ e $90,3 \%$ respectivamente). Pseudomo-nas aeruginosa foi analisada quanto ao seu perfil de sensibilidade a cefepima, piperacilinatazobactam, ceftazidima, meropenem, ciprofloxacino e aztreonam nas hemoculturas, amostras respiratórias e no total. Os resultados estão representados na figura 3. Verificou-se melhor perfil de sensibilidade da Pseudomonas aeruginosa à ceftazidima (79,2\%), seguido pela piperacilina-tazobactam (68,5\%), meropenem (58\%), cipro-floxacino $(57,8 \%)$, cefepima (56\%) e aztreonam (42,7\%). Essa tendência se manteve nos isolados de hemocultura e de amostras respiratórias. Nenhum antimicrobiano avaliado, exceto ceftazidima, apresentou atividade antipseudo-monas acima de $85 \%$ nas amostras estudadas. 
Figura 3: perfil de resistência do Pseudomonas aeruginosa aos diferentes antimicrobianos nos isolados de hemocultura, amostras respiratórias.

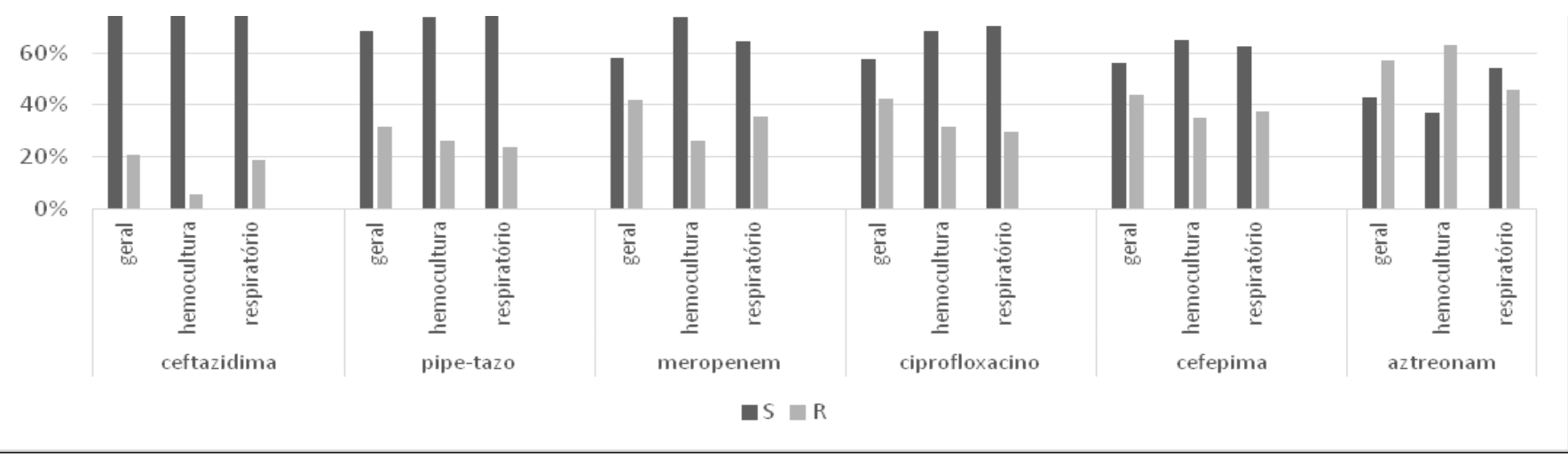

\section{DISCUSSÃO}

O Hospital de Clínicas é um hospital terciário vinculado à Universidade Federal do Paraná que conta com aproximadamente 600 leitos entre enfermarias e unidades de terapia intensiva. Por ser um hospital de alta complexidade, recebendo muitos pacientes provenientes de outras instituições de saúde, possui alta prevalência de germes multirresistentes, algumas das quais com comportamento endêmico.

Dentre as bactérias gram positivas, o MRSA representou $22 \%$ do total de isolados e $16 \%$ das hemoculturas, contrastando com os dados nacionais que mostram 30 a $60 \%$ de prevalência. O VRE representou $48,1 \%$ dos isolados de Enterococcus $s p$. Teve prevalência de $76,8 \%$ entre $E$. faecium e apenas $1,7 \%$ entre E. faecalis. Hemoculturas representaram $13 \%$ do total de isolados e VRE teve menor representatividade neste sítio $(9,5 \%)$

Os patógenos produtores de ESBL confirmaram ser destaque na epidemiologia hospitalar assim como em outros centros nacionais. Cepas de enterobactérias resistentes à cefepima representaram $41 \%$ do total dos isolados. Klebsiel-la pneumoniae foi o principal isolado em hemoculturas, onde apresentou $60,4 \%$ de resistência à cefalosporina de quarta geração

O tratamento empírico da infecção causada por Acinetobacter baumannii é um desafio para a equipe assistencial, tendo em vista as altas taxas de resistência à ampici-lina-sulbactam $(89,2 \%)$ e aos carbapenêmicos $(90,3 \%)$.

As opções terapêuticas tornam-se escassas e se limitam às polimixinas e tigeciclina.

Assim como visto na avaliação dos Acinetobacter baumanii, há também grande dificuldade na escolha empírica da terapia das infecções causadas por Pseudomonas aeruginosa, uma vez que os antimicrobianos avaliados por muitas vezes oferecem pouca frequência de atividade contra os isolados avaliados.

A ceftazidima foi o antibiótico de melhor espectro para cobertura da Pseudomonas aeruginosa, ocorrendo em $79,2 \%$ dentre o total, $94,4 \%$ entre as hemoculturas e $81,5 \%$ entre as amostras respiratórias. Porém, é pouco utilizada como terapia empírica, já que possui limitação para cobertura de

outros patógenos multirresistentes. Piperacilinatazobactam e cefepima são as drogas normalmente utilizadas com o objetivo de garantir cobertura contra este patógeno, porém apresentaram resistência acima do aceitável (>24\%). Cefepima teve pior cobertura em relação à piperacilina-sulbactam, sendo que a primeira apresentou resistência de $44 \%, 37,5 \%$ e $35 \%$ no total, nas hemoculturas e amostras respiratórias respectivamente contra $31,5 \%, 26,3 \%$ e $23,4 \%$ nas mesmas amostras testadas à segunda droga. A utilização do carbapenêmico com o intuito de garantir cobertura empírica para Pseudomonas aeruginosa deve ser revista, pois na amostra avaliada, $42 \%$ foram resistentes ao meropenem, sendo $26.3 \%$ em hemoculturas e $35.4 \%$ em amostras respiratórias. Cerca de metade das cepas são resistentes ao Aztreonam.

As amostras isoladas representam a somatória de todos os resultados e não necessariamente representam infecção, principalmente em relação aos sítios não estéreis (urina; amostras respiratórias) ou às bactérias colonizantes de pele, que podem contaminar amostra (Ex: Estafilococo não produtor de coagulase). Além disso, esses resultados não refletem as características de uma unidade específica e devem ser interpretados de acordo com as características locais de cada unidade em diferentes setores do hospital.

\section{CONCLUSÃO}




\section{REVISTA MÉDICA DA UFPR}

É muito importante a discussão e análise periódica do perfil de sensibilidade em um ambiente hospitalar, pois cada centro possui características específicas em relação a estrutura, equipe e perfil de pacientes que fa-zem com que seja um ambiente único, com uma identida-de própria. A prevalência de patógenos multirresistentes é alta no ambiente do Hospital de Clínicas. Entre os gram positivos, há menor prevalência de MRSA quando comparado com outros centros nacionais. Em relação aos Ente-rococcus $s p$, quase metade das cepas são VRE, sendo que essa proporção cai para perto de $10 \%$ quando avaliado somente as hemoculturas. Confirmou-se a alta prevalência de patógenos resistentes às cefalosporinas de quarta geração, levando a crer que há alta prevalência de patógenos produtores de ESBL. Klebsiella pneumoniae se destaca por ser a principal enterobactéria isolada em hemocultura e o que possui maior prevalência de cepas resistentes à cefepima.

O tratamento das infecções por Pseudomonas aeruginosa e Acinetobacter baumanii é um desafio, uma vez que a escolha de esquema antimicrobiano empírico é dificultado pelas elevadas taxas de resistência aos agentes comumente utilizados para tratamento desses agentes. Com relação a Pseudomonas $s p$, ceftazidima teve a melhor performance em relação à cobertura das cepas isoladas e deve ser considerada como uma das alternativas para a formação de esquema empírico.

Educação médica continuada e instituição de programa de uso racional de antimicrobianos são estratégias utilizadas para evitar uso desnecessário de antimicrobianos.

Tais medidas visam reduzir a emergência de germes multirresistentes e consequentemente a morbimortalidade das infecções nosocomiais.

\section{AGRADECIMENTOS}

Ao Serviço de Controle de Infecção Hospitalar ao disponibilizar os dados e ao Serviço de Informática, que possibilitou a avaliação dos resultados das culturas a partir dos dados do Sistema Interno Hospitalar.

\section{CONFLITOS DE INTERESSE}

Não houve conflito de interesses.

\section{REFERÊNCIAS BIBLIOGRÁFICAS}

1.Zarb P, Amadeo B, Muller A, Drapier N, Vankerckhoven V, Davey P, Goossens H; ESAC-3 Hospital Care Subproject Group.; Identification of targets for quality improvement in antimicrobial prescribing: the web based ESAC Point Preva-lence Survey 2009; J Antimicrob Chemother. 2011 Feb;66(2):443-9. doi: 10.1093/jac/dkq430. Epub 2010 Nov 17.

2.de Kraker ME, ET al.; Burden of antimicrobial resistance in European hospitals:excess mortality and length of hospitalstay associatedwith bloodstream infections due to Es-cherichia coli resistant to third - generation cephalosporins; J Antimicrob Chemother. 2011 Feb;66(2):398-407. doi: 10.1093/jac/dkq412. Epub 2010 Nov 23.

3. Kumar A, et al.; Duration of hypotension before initiation of effective antimicrobial therapy is the critical determinant of survival in human septic shock.; Crit Care Med. 2006 Jun;34(6):1589-96.

4.Jones RN.; Microbial etiologies of hospital-acquired bac-terial pneumonia and ventilator-associated bacterial pneu-monia; Clin Infect Dis. 2010 Aug 1;51 Suppl 1:S81-7. doi: 10.1086/653053.

5.Gales AC, Sader HS, Ribeiro J, Zoccoli C, Barth A, Pignatari AC.; Antimicrobial susceptibility of gram-positive bacteria isolated in Brazilian hospitals participat-ing in the SENTRY Program (2005-2008); Braz J Infect Dis. 2009 Apr;13(2):90-8.

6.Flávia Rossi; The Challenges of Antimicrobial Resistance in Brazil; Clinical Infectious Diseases 2011;52(9):1138-1143

7. Sader HS, Moet GJ, Jones RN.; Antimicrobial resistance among Gram-positive bacteria isolated in Latin American hospitals; J Chemother. 2009 Dec;21(6):611-20.

8.Ronald N. Master, Richard B. Clark, James A. Karlowsky, Julio Ramirez, Jose M. Bordon; Analysis of resistance, cross-resistance and antimicrobial combinations for Pseu-domonas aeruginosa isolates from 1997 to 2009; Interna-tional Journal of Antimicrobial Agents 38 (2011) 291- 295. 